\title{
A Practical Performance Comparison of Two Parallel Fast Fourier Transform Algorithms on Cluster of PCs
}

\author{
Kalim Qureshi* Non-member \\ Haroon Rashid** Non-member
}

In this paper, we present the performance analysis of two parallel Fast Fourier Transform algorithms, binary-exchange and transpose algorithms. These two algorithms were implemented and tested on a cluster of PCs using Message Passing Interface (MPI). The binary-exchange algorithm implementation was showing less processing and communication time than transpose algorithm.

Keywords : Parallel FFT, Tasks partitioning \& scheduling, Distributed system, Performance evaluation

\section{Introduction}

Fast Fourier Transform plays an important role in several scientific and technical applications. Some of these applications include Time Series and Wave Analysis, solving Linear Partial Differential Equations, Convolution, Digital Signal Processing and Image Filtering, etc. Hence, there has been a great interest in implementing FFT on parallel computers ${ }^{(1)}$.

\section{The Binary-exchange Algorithm}

We have $n$-point FFT, ranging from 16 to 4096 points, to be implemented on a cluster of PCs simulating a hypercube network with $p$-nodes, ranging from 2 to 16 nodes. We assume both $n$ and $p$ are power of two. We partition the input sequences into blocks of $(n / p)$ contiguous elements and assign one block to each processor $^{(2)(3)}$. For the simulated hypercube network, we assume $d$-dimensional cube ranging from 1 to 4 dimensions, knowing that $p=2^{d}$ and $n=2^{r}$ where $r=\log (n)$.

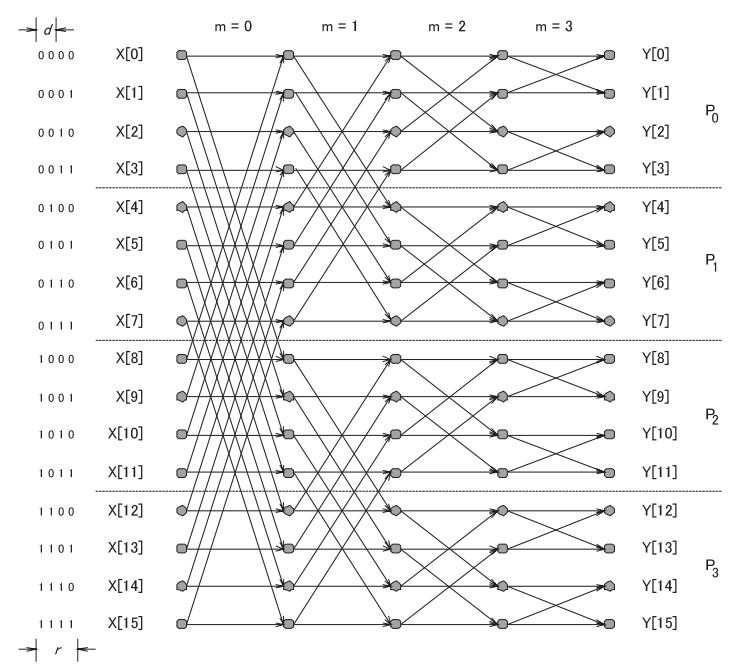

Fig. 2.1. The pattern of combination of elements of the input and the intermediate sequences during a 16-point unordered FFT computation

* King Fahd University of Petroleum \& Minerals, Dhahran, Saudi Arabia

** COMSATS Institute of Information Technology, Abbottabad, Pakistan
The elements with indices differing in their most significant bits are mapped onto different nodes. However, all elements with indices having the same $d$-most significant bits are mapped onto the same processor. This parallel FFT algorithm performs inter-process communication only during the first $d=\log (p)$ of a total $\log (n)$ iterations. For the rest of $\log (n)$ iterations, there is no inter-process communication ${ }^{(2)}$. During the communication operations, $n / p$ words of data are exchanged between nodes ${ }^{(3)}$.
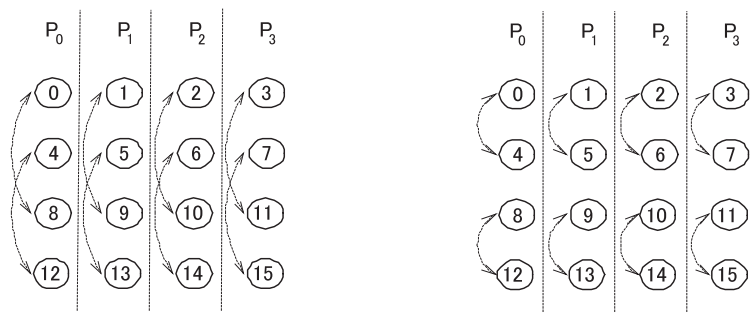

(a) Steps in phase 1 of the transpose algorithm (before transpose)
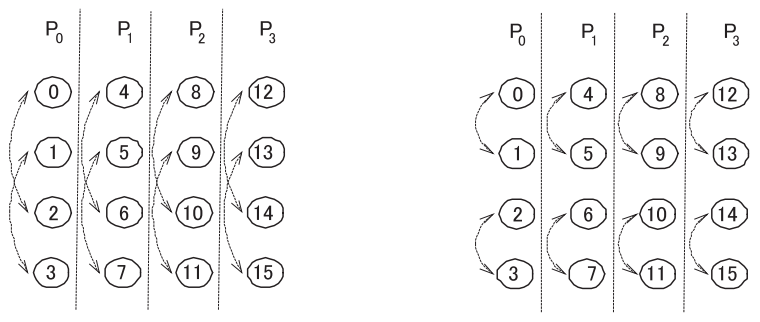

(b) Steps in phase 3 of the transpose algorithm (after transpose)

Fig. 2.2. The two-dimensional transpose algorithm for a 16-point FFT

2.1 The Transpose Algorithm Let the input sequence be arranged in a $\sqrt{n} \times \sqrt{n}$ two dimensional array in row major order. An ordered Fourier Transform of the input sequence can be obtained by performing an ordered radix-2 FFT over all the rows of this 2-D array followed by an ordered radix-2 FFT over all the columns $^{(4)}$. The row FFT corresponds to the first $\log n / 2$ 
iterations of the FFT over the entire input sequence and the column FFT corresponds to the remaining $\log n / 2$ iterations. In a parallel implementation, this $\sqrt{n} \times \sqrt{n}$ can be mapped on to $p$ nodes $(p \leq \sqrt{n})$ such that each processor stores $\sqrt{n} / p$ rows of the array ${ }^{(4)}$. Now the FFT over the rows can be performed without any inter-processor communication. After this step, the 2-D array is transposed and an FFT of all the rows of the transpose is computed. The only step that requires any inter-processor communication is transposing an $\sqrt{n} \times \sqrt{n}$ array on $p$ nodes. In general, the two-dimensional transpose algorithm works in three phases:

1) a $\sqrt{n}$-point FFT is computed for each column.

2) The array of data is transposed.

3) Identical to phase 1, and involves the computation of $\sqrt{n}$-point FFT for each column of the transposed array.

The figure below shows that the first and third phases of the algorithm don't require any inter-process communication. In both these phases, all $\sqrt{n}$ points for each column-wise FFT computation are available on the same processor. Only the second phase requires inter-process communication for transposing $\sqrt{n} \times \sqrt{n}$ matrix.

\section{Implementation}

3.1 Environment Setup The performance analysis was implemented and run on a cluster of PCs with the following hardware and software:

$\begin{array}{ll}\text { Hardware } & 16 \mathrm{PIII} \text { nodes } \\ \text { Processor Speed } & 1.0 \mathrm{GHz} \\ \text { Memory } & 2.0 \mathrm{~GB} / \text { node } \\ \text { Interconnect } & 100 \mathrm{Mb} / \mathrm{s} \text { Fast Ethernet } \\ \text { Operating System } & \text { Linux 2.4.9-31 } \\ \text { Parallel Environment } & \text { LAM MPI 6.5.6 }\end{array}$

Assumptions: During this experiment, we assume all our input sequences to be real numbers with no complex numbers and all operations are in real numbers. We also assume the complex root of unity $(W)$ equals to one all the way during the experiment.

\section{Results and Discussion}

4.1 Processing Time Overall, the binary exchange has less processing time when run on 2 to 16 nodes with varying $n$ size from 16 to 4096 points. However, difference is negligible for small FFT orders, $n \leq 512$ points. The performance difference between the two algorithms shows clearly for $n=4096$ where we can notice good scalability for both algorithms. The processing time decreases as we increase number of nodes. The measured results show that transpose algorithm has better scalability than binary-exchange.

4.2 Communication Time The communication time decreases as we increase number of nodes. When running on different nodes, Binary exchange has less varying communication time compared to that of the transpose algorithm where we see large difference between communication times when running on 8 , and 16 nodes for the same FFT order.
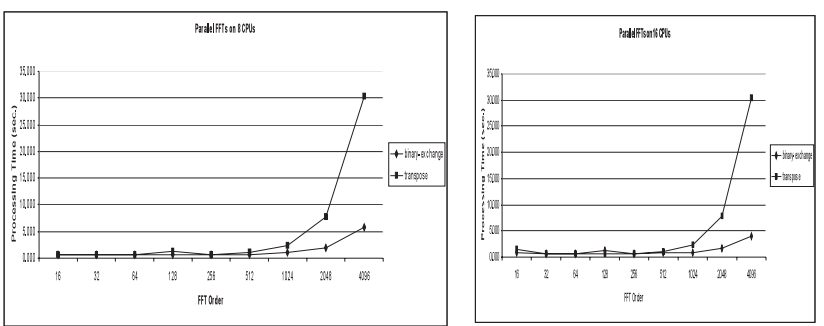

Fig. 4.1. FFT processing time on cluster of PCs 8 and 16
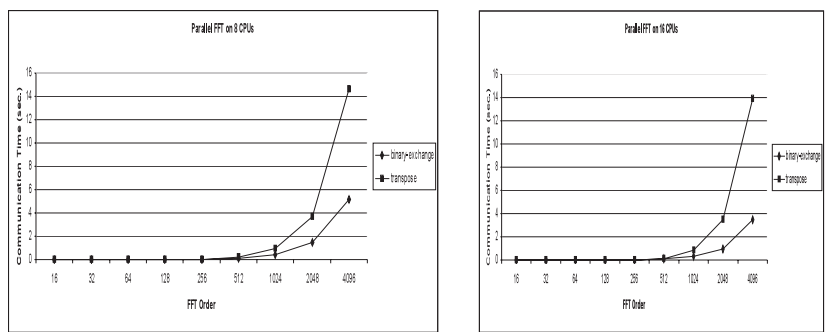

Fig. 4.2. Communication Time on 8 and 16 cluster of PCs

\section{Conclusion}

We have shown that on a PC cluster, binary-exchange algorithm has less processing and communication time than transpose algorithm. However, we anticipate that if we increase $n$ and increase $\mathrm{p}$, we may reach a point where transpose algorithm is much better than binary-exchange algorithm.

Future work will need to concentrate on finding the limit where transpose algorithm becomes better than binary-exchange and also find the scalability analysis when run on a large cluster $>16$ nodes. (Manuscript received April 1, 2004)

\section{References}

(1) A. Averbuch, E. Gabber, B. Gordissky, and Y. Medan : "A parallel FFT on an MIMD machine", Parallel Computing (2000)

(2) A. Norton and A. J. Silberger : "Parallelization and performance analysis of the Cooley-Tukey FFT algorithm for shared memory architectures", IEEE Transactions on Computers (1997)

(3) V. Kumar, A. Grama, A. Gupta, and G. Karypis : "Introduction to Parallel Computing, design and analysis of algorithms", Addison Wesley Higher Education (2002)

(4) A. Gupta and V. Kumar : "The Scalability of FFT on Parallel Computers", IEEE Transactions on Parallel and Distributed Systems (1996)

Kalim Qureshi (Non-member) He is an Assistant Professor in Department of Information and Computer Science at King Fahd University of Petroleum, Dhahran, Saudi Arabia. He is did his MS and Ph.D from Dept. of Computer Science and Systems Engg., Muroran Institute of Technology, Hokkaido, Japan in 1997 and 2000 respectively. He is a member of IEEE Computer Society. He can be contact by E-mail: qureshi@ccse.kupm.edu.sa.

Haroon Rashid (Non-member) is a director and faculty member of Department of Computer Science at COMSATS Institute of Information Technology, Abbottabad Campus, Pakistan. His research interests include parallel computing, distributed systems, high speed networks, multimedia, and network performance optimization. His E-mail: haroon@ciit.net.pk 